Recepción: 20 / 05/ 2018

Aceptación: 25 / 06 / 2018

Publicación: 01 / 08 / 2018

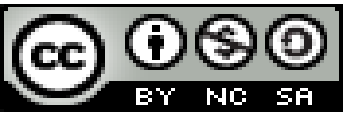

Ciencias Turísticas

Artículo Científico

\title{
Modelo de ruta turística patrimonial para impulsar bienes inmuebles patrimoniales culturales en el desarrollo turístico en el cantón Jipijapa, Ecuador
}

Heritage tourist route model to promote cultural heritage

real estate in the development tour in the

Jipijapa canton, Ecuador

Modelo de rotas turísticas patrocinadas para impulsionar o patrimônio cultural do patrimônio no desenvolvimento do turismo no cantão de Jipijapa, Equador

Shyla R. Orlando-Narváez I

shyla.orlando@unesum.edu.ec

Vanessa T. Santos-Moreira II

vanessa.santos@unesum.edu.ec

Manuel Castro-Priego III

manuel.castro@uah.es

Correspondencia: shyla.orlando@unesum.edu.ec

\footnotetext{
I. Docente Universidad Estatal del Sur de Manabí, Jipijapa, Ecuador.

II. Docente Universidad Estatal del Sur de Manabí, Jipijapa, Ecuador.

III. Docente de la Universidad de Alcalá, Madrid, España
} 


\section{Resumen}

La creación de la ruta turística patrimonial "Villa de Oro" se desarrolló mediante el enfoque exploratorio, que dio a conocer más sobre la zonificación turística de la ciudad de Jipijapa; y mediante la metodología descriptiva, la cual logró captar la situación real de Jipijapa a través de matrices de evaluación y análisis de la calidad de los servicios turísticos. Herramientas que fueron necesarias para el diseño de la ruta turística, que se elaboró bajo el modelo de Planificación Turística, Ordenación Territorial. Diseño realizado en conjunto con un modelo de interpretación del patrimonio cultural, el cual logrará afianzar la identidad cultural de la ciudad de Jipijapa. Aportando desde el ámbito turístico a la Declaratoria de los Bienes Inmuebles Patrimoniales Culturales Nacionales de la ciudad de Jipijapa, provincia de Manabí, Ecuador.

Palabras claves: Ruta turística patrimonial, zonificación turística, planificación turística, declaratoria de bienes patrimoniales, identidad cultural. 
Modelo de ruta turística patrimonial para impulsar bienes inmuebles patrimoniales culturales en el desarrollo turístico en el cantón Jipijapa, Ecuador

\section{Abstract}

The creation of the heritage touristic trail "Villa de Oro" was developed by means of the exploratory method, that made known more about the tourist zoning of the city of Jipijapa; and through the descriptive methodology, which managed to capture the real situation of Jipijapa through the use of matrix of evaluation and analysis of the quality of tourism services. Those tools were necessary for the design of the tourist trail that was elaborated under the model of Tourism Planning, Tourist Territorial Ordering. Design made in conjunction with a model of cultural heritage interpretation, which will strengthen the cultural identity of the city of Jipijapa. So, in that way the heritage tourism make an important contribution with the actual Declaration of Cultural Heritage of Ecuador.

Key words: Heritage tourist trail, tourist zoning, tourism planning, declaration of cultural heritage, cultural identity. 


\section{Introducción.}

En este artículo se argumentará acerca del proceso de creación de la Ruta Turística Patrimonial "Villa de Oro" como contribución a la declaratoria de bienes inmuebles patrimoniales culturales nacionales de la Ciudad de Jipijapa, Provincia de Manabí, Ecuador". El mismo comienza primero con el proceso de identificación de los recursos patrimoniales, los mismos que fueron oficialmente declarados en el Acuerdo Ministerial Nro. DM-2017-019 impulsado en el Gobierno Municipal actual.

A partir de la identificación de recursos patrimoniales se abre la etapa de la activación turística patrimonial que inicia a partir del desarrollo de una propuesta de ruta turística patrimonial como contribución a la declaratoria de bienes inmuebles patrimoniales culturales nacionales de la Ciudad de Jipijapa.

El estudio se desarrolla con planteamientos teóricos que realizaron reconocidos investigadores como Tomás López, Sandra Sánchez, Loreto Pérez y Juan Rengifo. Las fuentes utilizadas para argumentar y describir el proceso de identificación, y activación turística patrimonial, provienen de los estudios científicos realizados en turismo cultural, e itinerarios culturales, de notas de revistas y cartas internacionales publicadas por la Organización Mundial del Turismo (OMT), por la International Council on Monuments and Sites (ICOMOS) y de la experiencia en la gestión departamental del turismo y el patrimonio de los autores del presente artículo.

Se precisa además, la necesidad de avanzar en un proceso de planificación responsable en la activación turística patrimonial a partir de la propuesta de la ruta turística patrimonial. La preservación, la articulación con otras políticas regionales y nacionales, la participación de la sociedad local y el capital humano serán factores claves en esta planificación responsable, la que se espera pueda contribuir a diversificar la zona local. Solo de esta forma, como nos dice Jacqueline Plass, se 
Modelo de ruta turística patrimonial para impulsar bienes inmuebles patrimoniales culturales en el desarrollo turístico en el cantón Jipijapa, Ecuador

debe impulsar y desarrollar el turismo cultural en cuanto diversifica la oferta turística, genera nuevas oportunidades de negocios, equilibra la estacionalidad e incentiva el aumento del gasto, todo esto como parte de los desafíos que enfrenta la industria turística.

\section{Marco Teórico}

En este ítem se desarrollan y exponen los diferentes aspectos teóricos que encierra la obra, tratando de destacar aquellos que facilitaran la comprensión en relación al tema de investigación.

\section{Turismo Patrimonial}

De acuerdo con (ICOMOS, 1976), en sus escritos relativos al turismo cultural o patrimonial se afirma: Aquella forma de turismo que tiene por objeto, entre otros fines, el conocimiento de monumentos y sitios histórico-artísticos. Ejerce un efecto realmente positivo sobre éstos en tanto en cuanto contribuye para satisfacer sus propios fines a su mantenimiento y su protección. Esta forma de turismo justifica, de hecho, los esfuerzos que tal mantenimiento y protección exigen de la comunidad humana, debido a los beneficios socio - culturales y económicos que comporta para toda la población implicada.

Acorde a lo expresado en esta revista podemos decir que en realidad, el turismo cultural es una tipología de turismo que se enfoca principalmente en la visita a diferentes lugares de interés cultural en una ciudad de destino cualquiera, esto con el fin de conocer su historia, aprender sobre su cultura, disfrutar de su patrimonio y del paisaje, es decir que el turismo cultural genera placer pero enriquece de conocimiento durante nuestro tiempo de ocio. 


\section{Importancia del turismo patrimonial}

Según un informe de la Subsecretaria de Turismo se expresa "la Organización Mundial de Turismo (OMT) señala que el turismo cultural representa sobre el $40 \%$ de las llegadas internacionales en el mundo y se ha transformado en un importante motor del turismo interno" (Plass W., 2012).

De acuerdo con (Plass W., 2012), en su informe Importancia del Turismo Patrimonial expresa: La cultura y el patrimonio constituyen motivadores de elección de destinos tan relevantes como la naturaleza, aventura y deportes, salud y bienestar, la vida urbana o la experiencia gastronómica. Debemos impulsar y desarrollar el turismo cultural en cuanto diversifica la oferta turística, genera nuevas oportunidades de negocio, equilibra la estacionalidad e incentiva el aumento del gasto, todo esto como parte de los desafíos que enfrenta la industria turística.

La Organización Mundial de Turismo (OMT, 2013), expresa lo siguiente: El turismo cultural puede generar nuevas oportunidades de empleo, atenuar la pobreza, frenar el éxodo rural entre los jóvenes y los subempleados así como cultivar un sentimiento de orgullo entre los miembros de las comunidades. El turismo, además, ofrece también un poderoso incentivo para conservar y potenciar el patrimonio cultural inmaterial, ya que los ingresos que genera pueden reconducirse hacia iniciativas que ayuden a su vez a su supervivencia a largo plazo.

Al analizar las rutas patrimoniales tenemos que (López-Guzmán, Tomás y Sánchez, Sandra, 2008) señalan: Estos recorridos turísticos, que toman distintas denominaciones tales como corredores, rutas, circuitos, caminos o itinerarios, tratan de diferenciarse de sus competidores resaltando determinados recursos que están presentes en el territorio o que son imaginados y creados artificialmente. En el primer caso se invita al visitante a recorrer un periplo en el que predomina una determinada categoría patrimonial, ya sean manifestaciones culturales, testimonios del pasado 
Modelo de ruta turística patrimonial para impulsar bienes inmuebles patrimoniales culturales en el desarrollo turístico en el cantón Jipijapa, Ecuador

arqueológico o histórico, patrimonio artístico, industrial o espacios naturales. Ejemplos de estos productos serían las rutas gastronómicas y enológicas, que basan su oferta en el aprovechamiento turístico de los recursos agropecuarios de un territorio.

Por su parte (Inglis, David y Holmes, Mary, 2003), al estudiar este tipo de rutas indican: Otros trazados obedecen a un diseño basado en leyendas y mitos -casi siempre adaptados muy libremente y con una buena dosis de elucubración histórica- que trata de inducir al turista a viajar por sitios particulares de los que se dice que poseen un encantamiento que trasciende. De estos recorridos se suele destacar la presencia de restos prehistóricos (dólmenes, necrópolis, petroglifos...) y medievales (fortalezas, ermitas, iglesias...) junto con accidentes geológicos llamativos a los que se atribuyen propiedades curativas y/o mágicas (aguas, barros, plantas medicinales...), así como la celebración de rituales perdidos o vigentes pero presentados como atávicos. Los espacios que recorren estas rutas son mostrados como insólitos y cargados de leyendas que refieren a acontecimientos, personajes históricos e incluso fantásticos (templarios, duendes, magos, hadas, gigantes, fantasmas, brujas...) combinados a veces con interpretaciones esotéricas sobre las propiedades del lugar. Ley de Patrimonio Cultural del Ecuador

De acuerdo con la Ley de Patrimonio Cultural del Ecuador, aprobada el 19 de noviembre del 2004, en su artículo 7 se tipifican las categorías o tipos de bienes calificados como patrimonio cultural estadal sujetos de tutela y protección especial, en esta se establece:

Art. 7.- Declárense bienes pertenecientes al Patrimonio Cultural del Estado los comprendidos en las siguientes categorías:

a) Los monumentos arqueológicos muebles e inmuebles, tales como: objetos de cerámica, metal, piedra o cualquier otro material pertenecientes a la época prehispánica y colonial; ruinas de 
fortificaciones, edificaciones, cementerios y yacimientos arqueológicos en general; así como restos humanos, de la flora y de la fauna, relacionados con las mismas épocas;

b) Los templos, conventos, capillas y otros edificios que hubieren sido construidos durante la Colonia; las pinturas, esculturas, tallas, objetos de orfebrería, cerámica, etc., pertenecientes a la misma época; ....omissis......

\section{Historia de Jipijapa}

De acuerdo con (Gobierno Provincial de Manabí, 2017), en su página Web describe la historia de Jipijapa en los términos siguientes:

El cantón Jipijapa tiene sus raíces prehispánicas, fue conquistado por Pedro de Alvarado, sometiéndole a este pueblo guerrero por la fuerza. El nombre de Jipijapa no se conoce a ciencia cierta de donde proviene se dice que viene de un Cacique llamado Xipixapa o Xipescape que en idioma nativo significa sube y baja por tierra alta y baja. Según la enciclopedia del mundo, "DURVAN", dice que "Jipijapa, faja fina y flexible sacada de la hoja del bombonaje y que se usa en diversos lugares de América Meridional para tejer petacas o sombreros.

Las 9 parcialidades indígenas que conformaban Jipijapa en vista de haber sufrido saqueos, incendios protagonizados por los españoles y piratas, denunciaron estos atropellos ante el Presidente de la Real Audiencia de Quito Don Fernando de Santillán, quién delegó al visitador Don Bernardo de Loaysa para que escuchando a los reclamantes arbitre las medidas necesarias para favorecerlos y lograr el renacimiento de la paz y la tranquilidad en estas comarcas, y se funda San Lorenzo de Jipijapa el 10 de Agosto de 1565 en lo que hoy es Sancán. El 8 de enero de 1822, se instaló el Primer Cabildo de Jipijapa. 
Modelo de ruta turística patrimonial para impulsar bienes inmuebles patrimoniales culturales en el desarrollo turístico en el cantón Jipijapa, Ecuador

En la vida republicana hasta la actualidad el cantón Jipijapa se ha ido desarrollando, especialmente en la agricultura convirtiéndose en el primer exportador de café por lo que se le conoce con el nombre "La Sultana del Café"; los bajos precios internacionales del café, las plantaciones cafeteras antiguas y su baja producción terminaron con esta “Época de Oro”. En la actualidad se cuenta con importantes instituciones que contribuyen al desarrollo de Jipijapa como es la Junta de Recursos Hidráulicos, la UNESUM, entre otras.

En 1534 en la conquista española Pedro de Alvarado en su marcha hacia la conquista del Reino de Quito con su ejército invasor asaltó por sorpresa al pueblo de Jipijapa, saqueándolo llevando inmensas riquezas como oro, plata, esmeralda, armadura y vestido de planchas. Tanta riqueza lo deslumbró y lo denominó "Pueblo de oro".

\section{Oferta y demanda turística de Jipijapa}

La oferta cultural del cantón Jipijapa se encuentra descrita en el Acuerdo Ministerial Nro. DM2017-019 que en su Art. 1, establece:

Art. 1.- Declara como bienes del patrimonio cultural nacional, a los diecisiete (17) inmuebles, un (1) conjunto urbano y dos (2) espacios públicos localizados en el cantón Jipijapa (provincia de Manabí), detallados en el expediente técnico de inventario elaborados por el Instituto Nacional de Patrimonio Cultural, los cuales se constituyen en documentos habilitantes de este Acuerdo Ministerial.

\section{Marco Metodológico}

Para el diseño de la ruta turística patrimonial "Villa de Oro" de la ciudad de Jipijapa se emplearon varios métodos, el primer método aplicado fue el exploratorio, el cual se utiliza cuando el tema o asunto es nuevo y cuando los datos son difíciles de recopilar (Ander-Egg, 1993); igualmente, 
se utilizó el método descriptivo, el cual tiene como objetivo describir situaciones y fenómenos, midiendo variables en forma independiente (Fassio, A.; Pascual, L., 2015). Para ello se realizan faenas de campo con miras a la recolección de información general, posterior a ello se efectúa tratamiento de los datos a través de trabajos de gabinete consistentes en procesamiento, análisis y presentación de resultados, y finalmente diseñar la ruta turística sobre la base de las tareas previamente ejecutadas.

\section{Trabajo de campo, obtención de información in situ:}

El trabajo de campo se divide en dos fases, la primera referida a la búsqueda de información documental disponible ante instituciones u organismos oficiales, además de la realización de entrevistas con las comunidades, personas propietarias de los bienes patrimoniales, cronistas de los sitios históricos y turistas visitantes de los mismos.

Adicionalmente se evalúa el conjunto de bienes a través del recorrido personal a sus instalaciones y edificaciones, valorando condiciones generales, localización, estado de conservación, accesibilidad, servicios conexos, entorno ambiental asociado, entre otros aspectos de interés general. Se precisa su localización en planos y mapas de la ciudad con miras al diseño de las rutas turísticas, adicionalmente se hace reseña fotográfica de cada bien inventariado.

Trabajo de gabinete, procesamiento de información:

Una vez obtenida la información documental y físico-ambiental de los bienes patrimoniales, se procede a su tratamiento, caracterización e inventario. Se diseñan fichas técnicas que contengan información general del patrimonio (Casas, iglesias, edificaciones históricas, etc.) a ser incorporado en la ruta turística cultural, en ellas se incorpora: época de construcción, categoría de la valoración, 
Modelo de ruta turística patrimonial para impulsar bienes inmuebles patrimoniales culturales en el desarrollo turístico en el cantón Jipijapa, Ecuador

valoración, criterio de valoración, grado de protección, descripción y observaciones generales, además de una reseña fotográfica de la edificación correspondiente y su respectiva localización geográfica.

Propuesta de resultados, diseño de la ruta turística:

La concreción del trabajo investigativo es el diseño de la ruta turística con base al análisis de la calidad de los servicios turísticos y zonificación turística (Mackinnon, Child \& Thorsell, 1996). Se trata de una propuesta cartográfica donde se localice con la debida precisión y detalle los iconos o patrimonios históricos inventariados, a los fines de promover y facilitar su accesibilidad y visitas por parte de turistas nacionales y extranjeros.

\section{Presentación de resultados}

A partir de la aplicación de los diferentes métodos y de la ejecución del trabajo de búsqueda de información, procesamiento y análisis de datos se presentan los resultados de la investigación.

\section{Inventario del patrimonio cultural}

Con base a los métodos exploratorios y descriptivos desarrollados como complemento a la revisión documental y los trabajos de campo, a continuación se presentan algunos de los bienes culturales inventariados, donde se puede apreciar el diseño de la ficha técnica correspondiente, observándose el contenido e información general que la misma contiene.

Es importante resalta que el total de bienes inventariados y estudiados s e presentan en la Figura 9, denominada: Cuadro de bienes inmuebles incorporados a la ruta turística patrimonial "Villa de Oro". 
Shyla R. Orlando-Narváez, Vanessa T Santos-Moreira, Manuel Castro-Priego

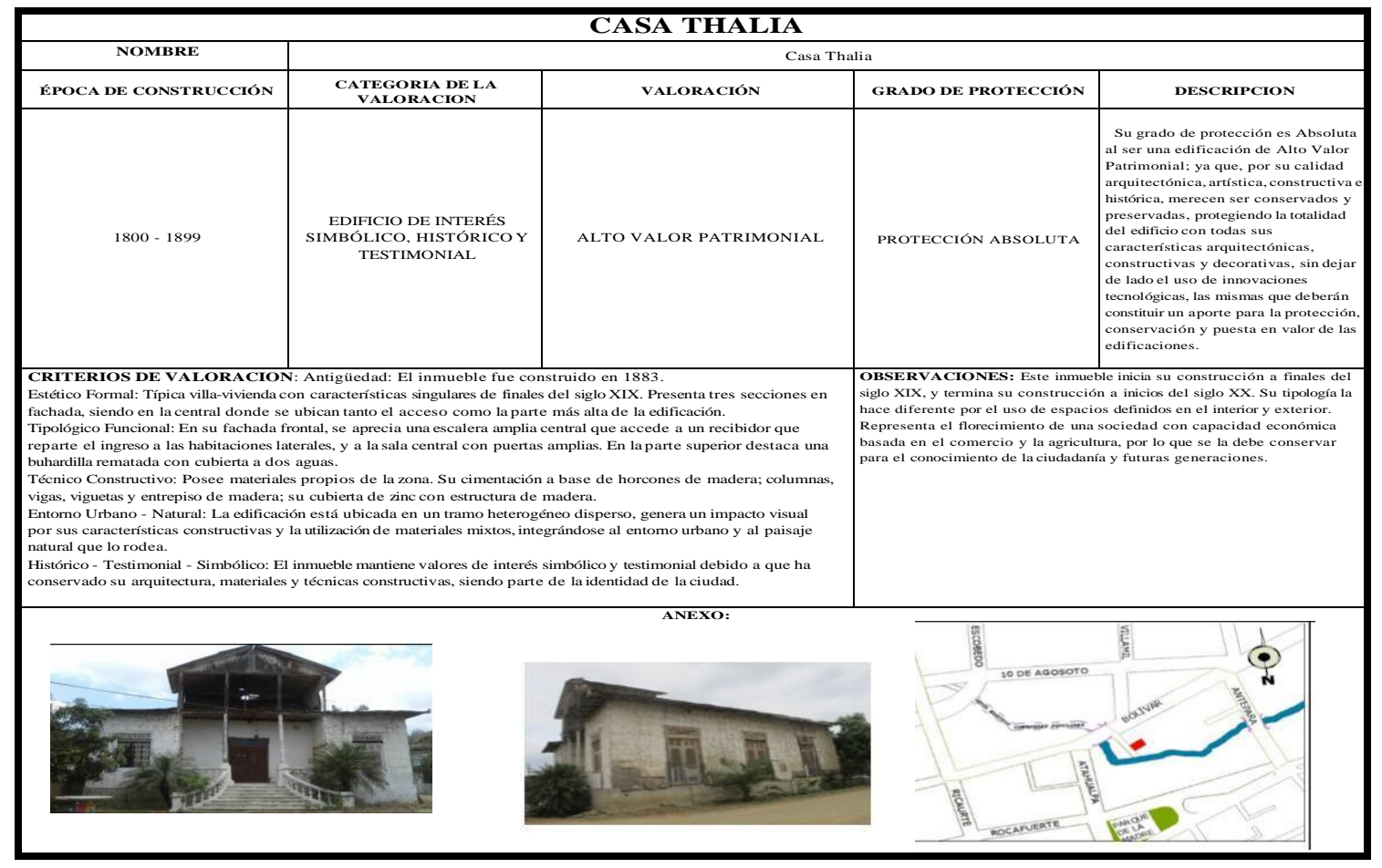

Figura 1. Ficha de inventario del patrimonio cultural "Casa Thalia".

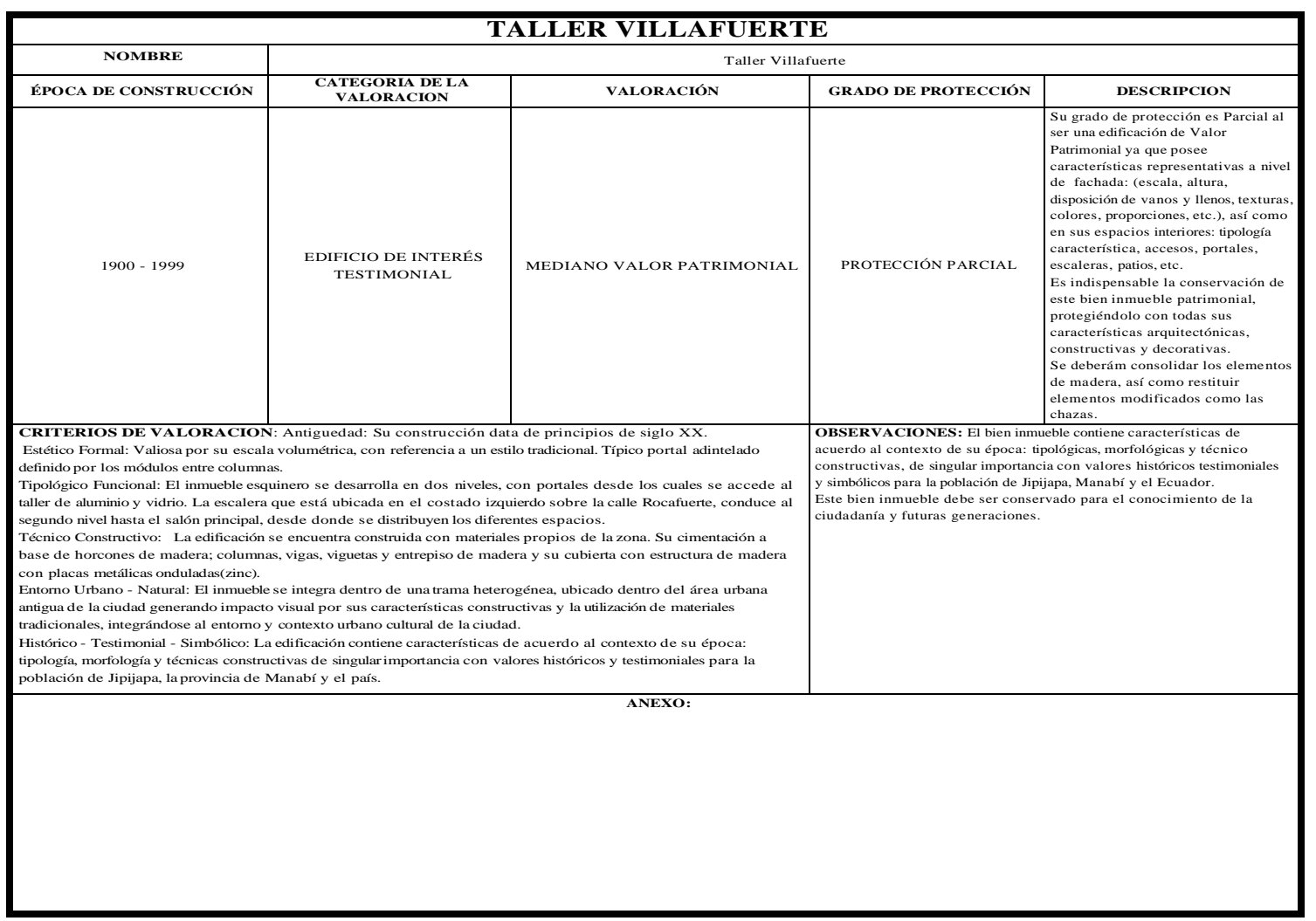

Figura 2. Ficha de inventario del patrimonio cultural "Taller Villafuerte". 
Modelo de ruta turística patrimonial para impulsar bienes inmuebles patrimoniales culturales en el desarrollo turístico en el cantón Jipijapa, Ecuador

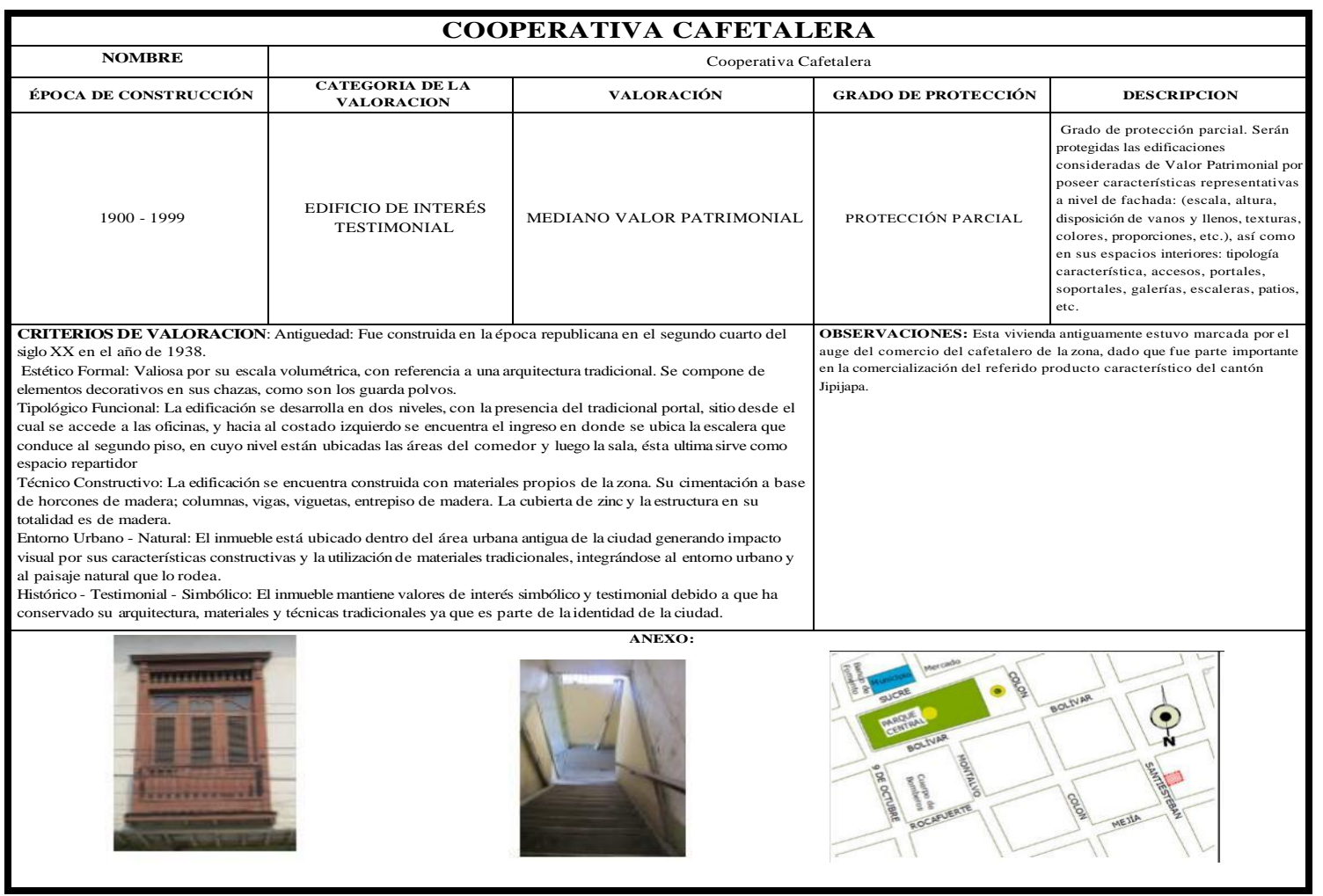

Figura 3. Ficha de inventario del patrimonio cultural "Cooperativa Cafetalera". 
Shyla R. Orlando-Narváez, Vanessa T Santos-Moreira, Manuel Castro-Priego

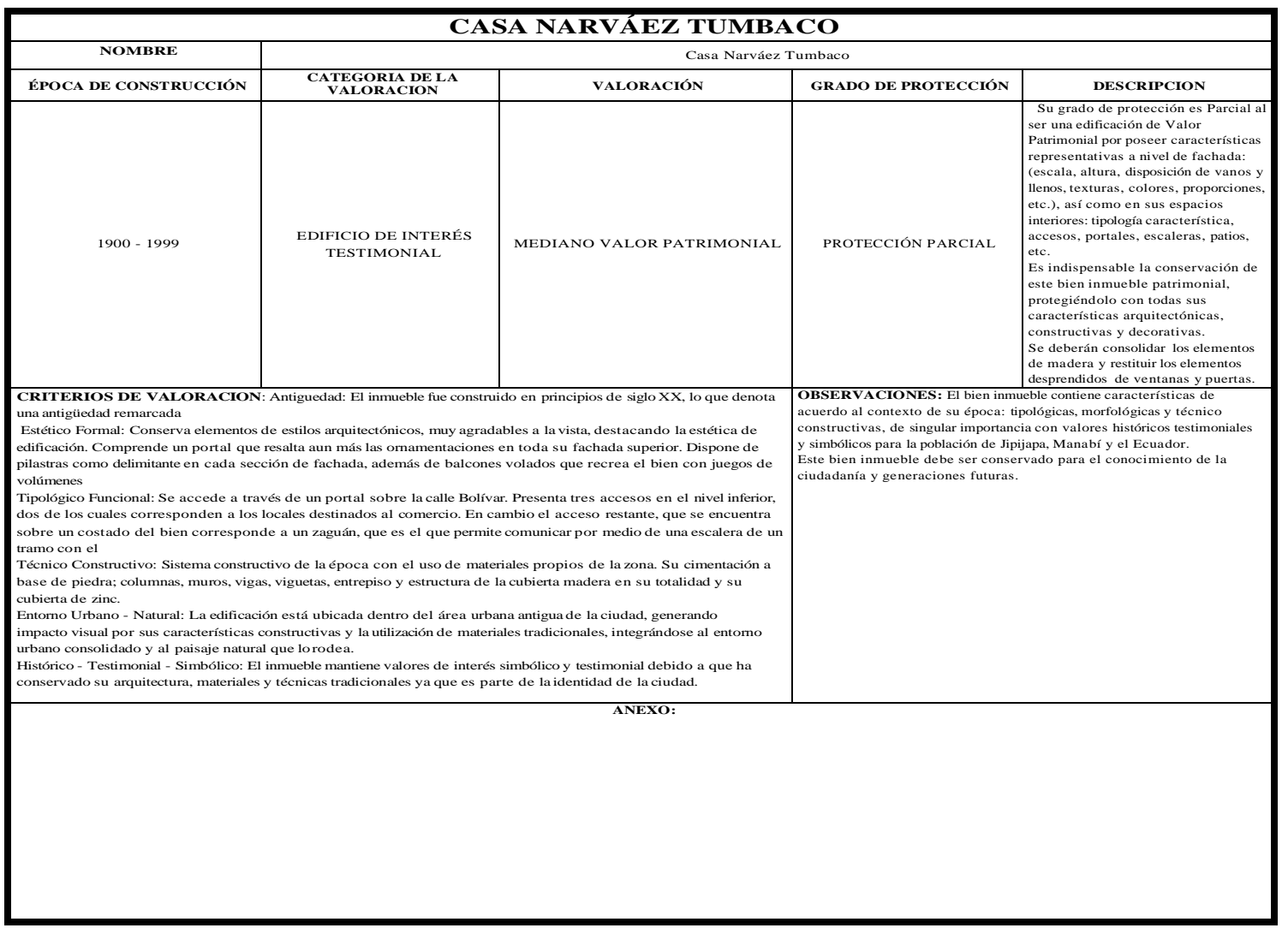

Figura 4. Ficha de inventario del patrimonio cultural "Casa Narváez Tumbaco". 
Modelo de ruta turística patrimonial para impulsar bienes inmuebles patrimoniales culturales en el desarrollo turístico en el cantón Jipijapa, Ecuador

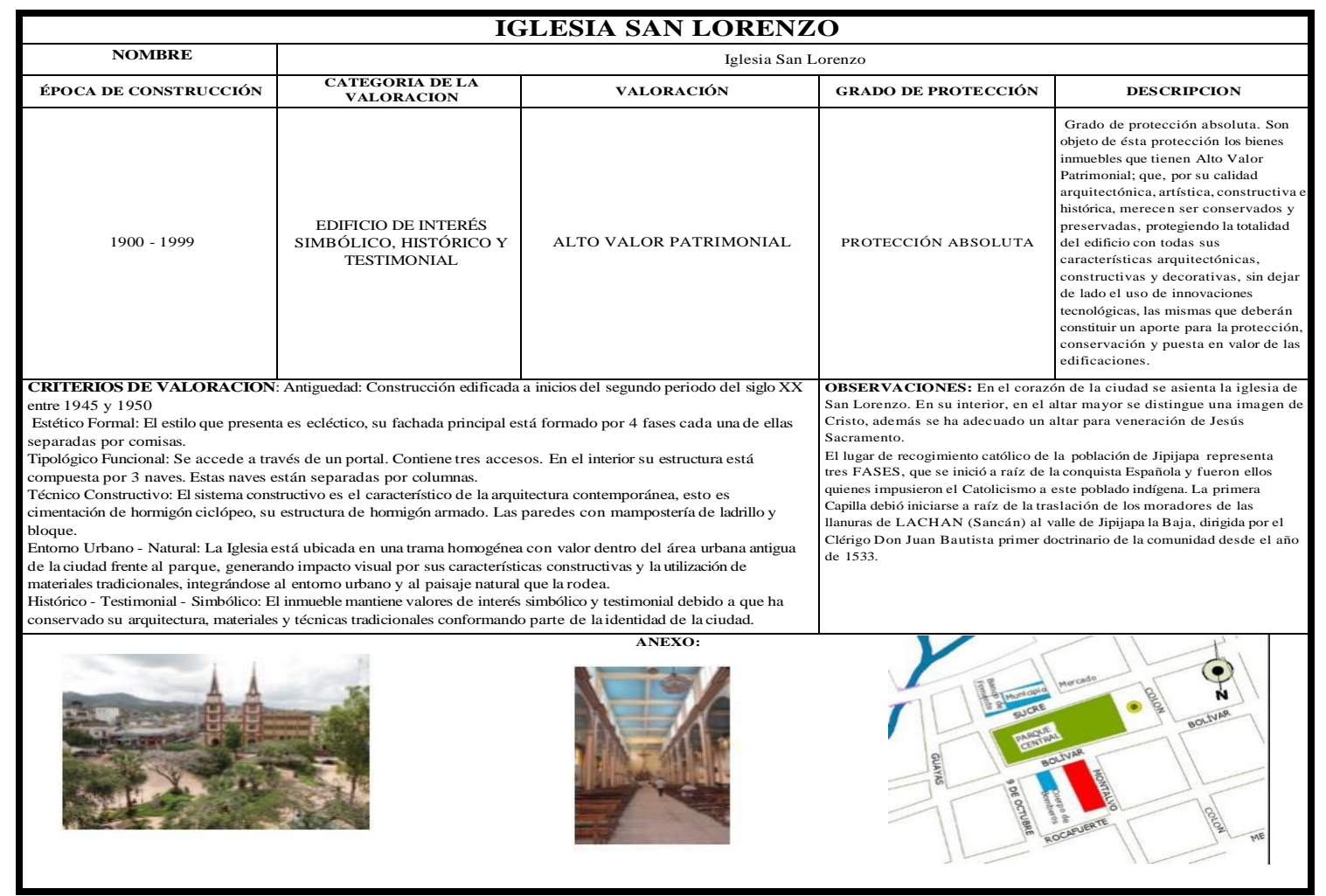

Figura 5. Ficha de inventario del patrimonio cultural "Iglesia San Lorenzo". 
Shyla R. Orlando-Narváez, Vanessa T Santos-Moreira, Manuel Castro-Priego

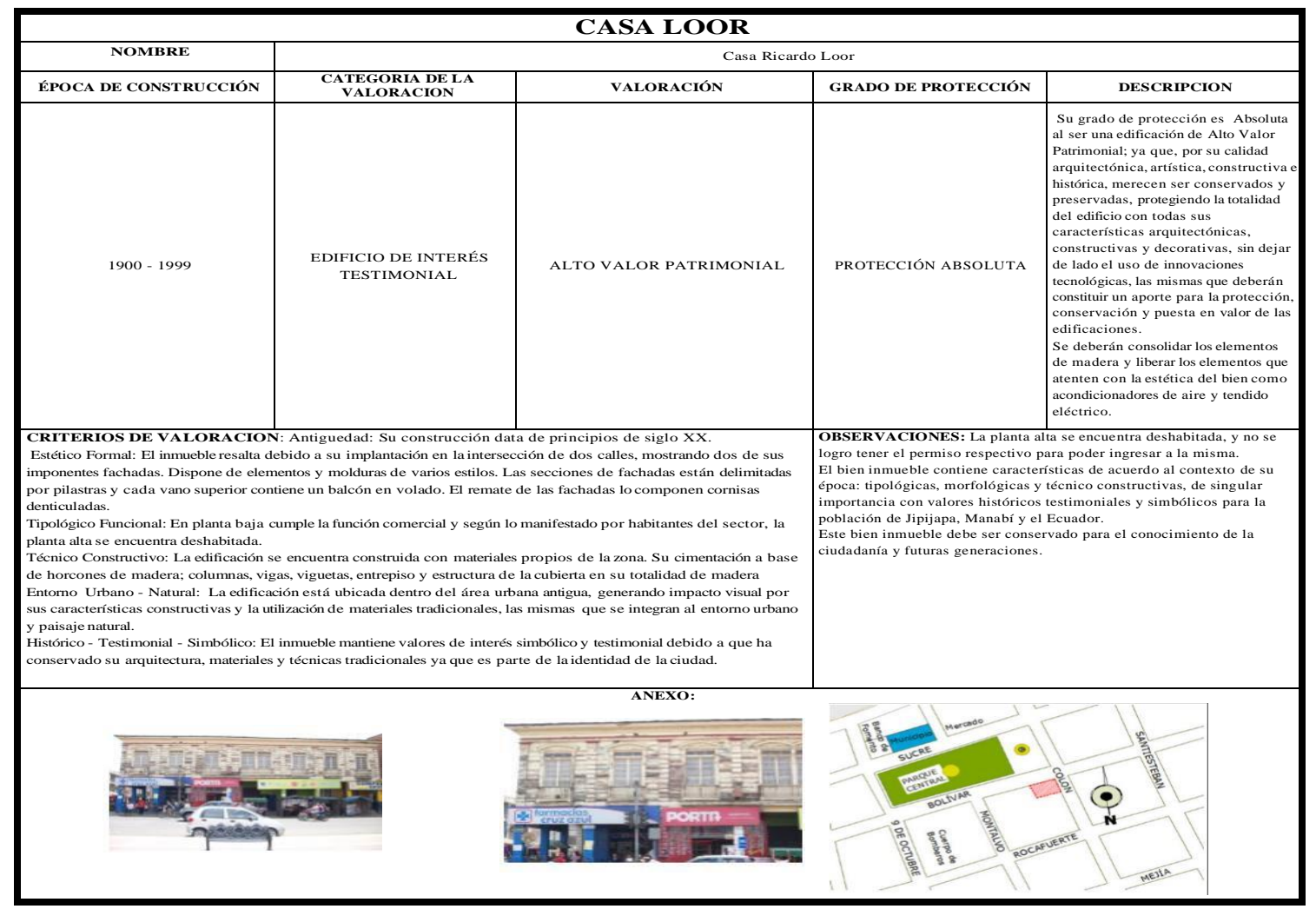

Figura 6. Ficha de inventario del patrimonio cultural "Casa Loor".

\section{Resultados}

Con respecto al análisis de la planta turística de la cabecera cantonal de Jipijapa, se realizaron varias visitas in situ de los principales establecimientos de alojamiento, restauración, agencias de viajes, servicios de transportación terrestre y centros turísticos ubicados dentro del perímetro de estudio. Se pudieron evidenciar los siguientes puntos:

-Elementos folclóricos y culturales con un alto grado de potencialidad a desarrollar.

- Cuenta con elementos lo suficientemente importantes para potencializar su patrimonio históricoartístico, arqueológico, artesanal y gastronómico.

-Trabajadores del turismo muy poco capacitados. 
Modelo de ruta turística patrimonial para impulsar bienes inmuebles patrimoniales culturales en el desarrollo turístico en el cantón Jipijapa, Ecuador

- No existe un plan de calidad y servicio al cliente implantado en la ciudad.

- No existe hotelería de calidad con servicios de hoteles de 4 o 5 estrellas.

-Establecimientos de alojamiento poco equipados.

- Gran variedad de atractivos turísticos inventariados por el Ministerio de Turismo de Ecuador.

-Patrimonio arquitectónico necesita un plan de rescate y mejoramiento.

- No existe un plan de turismo para la ciudad.

•Escasa publicidad y promoción de la ciudad en el mercado nacional e internacional.

-Buen servicio de transporte público (taxis y colectivos en buen estado y con precios bastante asequibles)

-Servicio de gastronomía con precios muy económicos.

No existe un perfil definido de la actual demanda turística de Jipijapa.

Al realizar el inventario de los bienes turísticos y efectuados su diagnóstico general, se procedió a diseñar el mapeo de la zonificación turística de la cabecera cantonal de Jipijapa. Dicha zonificación estuvo basada en el inventario realizado y en los criterios de la delimitación actual de las principales zonas turísticas establecidas por la Unión Internacional para la Conservación de la Naturaleza (UICN) (Mackinnon, Child \& Thorsell, 1996). El proceso de evaluación de campo y posterior mapeo arrojó como resultado los sitios turísticos mostrados en la Figura 7. 
Shyla R. Orlando-Narváez, Vanessa T Santos-Moreira, Manuel Castro-Priego

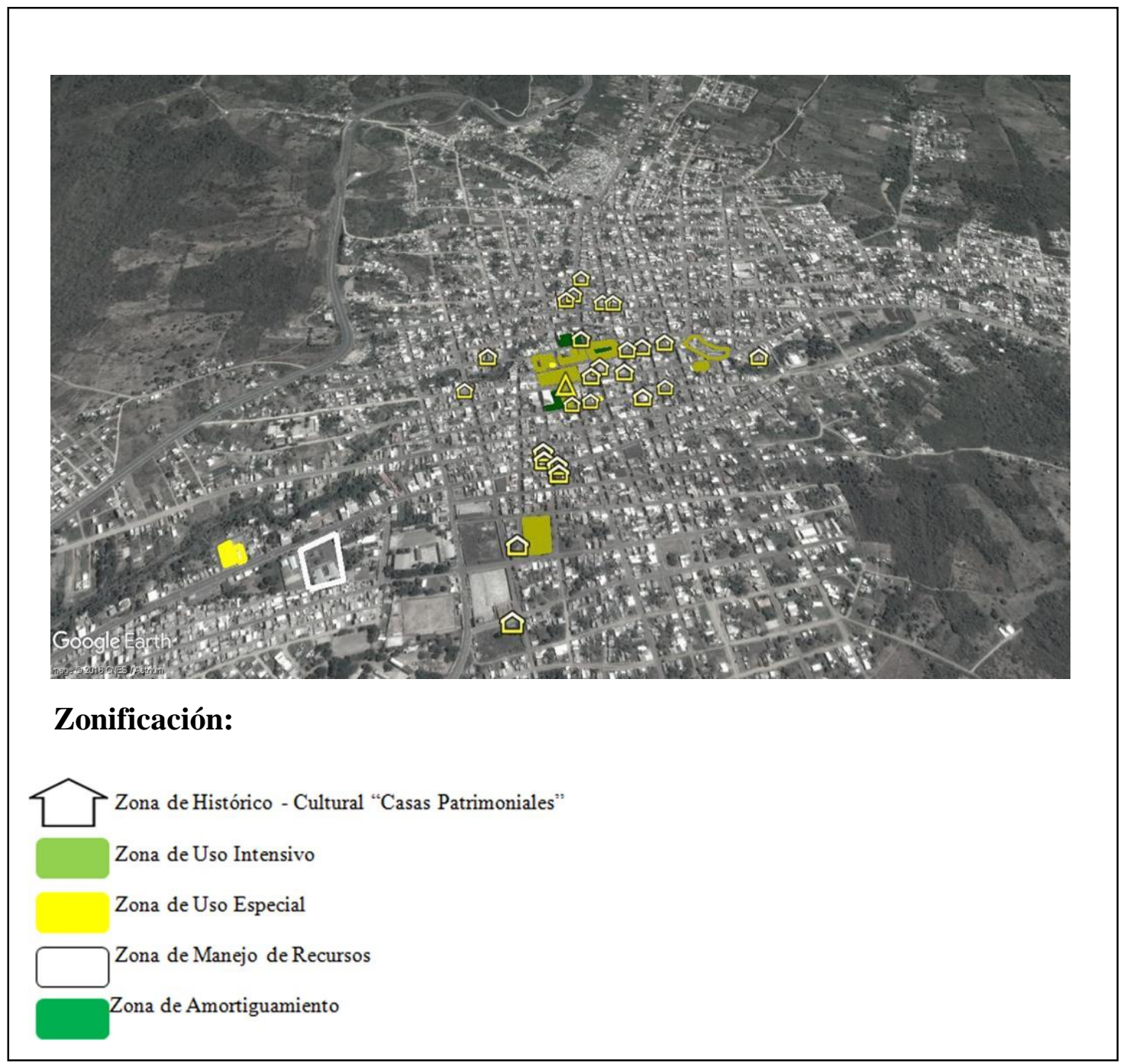

Figura 7. Zonificación turística de la cabecera cantonal de Jipijapa.

Dentro de la zonificación turística de la cabecera cantonal de Jipijapa se encontraron 5 zonas delimitadas bajo el criterio de la Zonificación general, las cuales son las siguientes:

- Zona de uso Intensivo-Extensivo

- Zona Histórica Cultural

- Zona de Manejo de Recursos 
Modelo de ruta turística patrimonial para impulsar bienes inmuebles patrimoniales culturales en el desarrollo turístico en el cantón Jipijapa, Ecuador

- $\quad$ Zona de uso especial (administración)

- Zona de Amortiguamiento

Al establecer una zonificación turística se podrá asegurar que las visitas ocurran en lugares y en formas que están dentro de la capacidad de manejo tanto en lugares de uso público como en lugares de uso turístico deseado. Así también, la zonificación turística de la cabecera cantonal de Jipijapa ayudará a proveer oportunidades para poder desarrollar más de un tipo de experiencia turística; mediante la identificación y agrupación de áreas con potencial turístico ubicados dentro del perímetro de estudio para de esa forma desarrollar futuras rutas turísticas. En el caso particular de Jipijapa se determinó que el producto turístico que mejor se adapta a la actual declaratoria como "Bienes Pertenecientes al Patrimonio Cultural Nacional de Ecuador a los 17 Inmuebles, 1 Conjunto Urbano y 2 Espacios Públicos localizados en la ciudad de Jipijapa, Provincia de Manabí”, expedido bajo el Acuerdo Ministerial Nro. DM - 2017 - 019(Ministerio de Cultura y Patrimonio de Ecuador, 2017) es el diseño de una ruta turística patrimonial.

La ruta turística patrimonial "Villa de Oro" estará enmarcada bajo lo indicado en el artículo 2 del Acuerdo Ministerial Nro. DM - 2017 -019, donde expresa lo siguiente: “Encargar la ejecución del presente Acuerdo Ministerial al Gobierno Descentralizado del cantón Jipijapa y al Instituto Nacional de Patrimonio Cultural, entidades que en el ejercicio de sus competencias, deberán cumplir con las disposiciones legales y reglamentarias de la materia, para emprender las acciones administrativas que garanticen el mantenimiento, conservación y difusión del conjunto de bienes del patrimonio cultural nacional descritos" (Ministerio de Cultura y Patrimonio de Ecuador, 2017). Para lograr tal cometido, luego de obtener los respectivos permisos de parte de los dueños de los bienes inmuebles, se establecieron un total de quince Bienes Inmuebles Patrimoniales para que compongan el total de 
atractivos turísticos a visitar dentro de la ruta turística patrimonial "Villa de Oro". Siendo estos los mostrados en la Figura 8.

Se determinó “Villa de Oro”, como el nombre de la ruta turística, ya que Jipijapa fue conocida por un tiempo con tal denominación, debido a las riquezas que encontraron los colonizadores cuando incursionaron en la zona, en el año 1534, comandados por Pedro de Alvarado (Toala, M. 2012). Siendo una ruta donde se va a resaltar de forma casi absoluta su riqueza arquitectónica, es pertinente recurrir a dicha denominación. Apelativo que va acorde al legado europeo que vivió en su momento en dicho cantón, el cual se logra plasmar en sus patrimonios arquitectónicos. La ruta tendrá una longitud de aproximadamente $2,25 \mathrm{~km}$.

\begin{tabular}{|c|c|c|}
\hline $\begin{array}{c}\text { NÚMERO DE ATRACTIVO } \\
\text { TURÍSTICO }\end{array}$ & $\begin{array}{l}\text { BIEN INMUEBLE } \\
\text { PATRIMONIAL }\end{array}$ & DIRECCION \\
\hline 1 & THALIA & Bolívar y Antepara \\
\hline 2 & HEREDEROS MATUTE & $\begin{array}{l}\text { Sucre entre Ricaurte y Víctor } \\
\text { Manuel Rendón }\end{array}$ \\
\hline 3 & $\begin{array}{l}\text { HEREDEROS ZAVALA - } \\
\text { BAQUE }\end{array}$ & Sucre y Víctor Manuel Rendón \\
\hline 4 & TALLER VILLAFUERTE & $\begin{array}{l}\text { Rocafuerte y Víctor Manuel } \\
\text { Rendón }\end{array}$ \\
\hline 5 & COOP. CAFETALERA & Santiesteban y Rocafuerte \\
\hline 6 & NARVAEZ TUMBACO & $\begin{array}{l}\text { Rocafuerte entre Montalvo y } \\
\text { Colon }\end{array}$ \\
\hline 7 & PIONCE MUÑIZ & $\begin{array}{c}9 \text { de Octubre entre Alejo } \\
\text { Lascano y Avilés }\end{array}$ \\
\hline 8 & IGLESIA MERCEDARIA & Bolívar y Montalvo \\
\hline 9 & RICARDO LOOR & Bolívar y Colon \\
\hline 10 & NIETO ZAVALA & Bolívar y Colon \\
\hline 11 & BUSTAMANTE & Santiesteban y Bolívar \\
\hline 12 & $\begin{array}{l}\text { HEREDEDOS ZAVALA - } \\
\text { GONZALEZ }\end{array}$ & Sucre y Santiesteban \\
\hline 13 & IDA CASTILLO PARRALES & $\begin{array}{l}\text { Parrales y Guale entre Colon y } \\
\text { Montalvo }\end{array}$ \\
\hline 14 & VILLACRESES & $\begin{array}{c}\text { Colon / Noboa y Parrales y } \\
\text { Guale }\end{array}$ \\
\hline 15 & GUARANDA & Parrales Iguale y Santiesteban \\
\hline
\end{tabular}

Figura 8. Cuadro de bienes inmuebles incorporados a la ruta turística patrimonial "Villa de Oro". 
Modelo de ruta turística patrimonial para impulsar bienes inmuebles patrimoniales culturales en el desarrollo turístico en el cantón Jipijapa, Ecuador

La circulación de la ruta turística "Villa de Oro" está estipulada a ser realizada en automóvil y/o a pie. Como atractivos turísticos culturales del cantón Jipijapa que acompañen al conjunto de bienes inmuebles patrimoniales. Se destaca demostraciones in situ del empleo de la caña guadua como elemento constructivo en la costa ecuatoriana. Así mismo, el Museo Arqueológico de la ciudad de Jipijapa servirá como atractivo complementario a la principal oferta de la ruta turística.

La recolección de información, tales como: Archivos históricos de la ciudad de Jipijapa y de los bienes inmuebles patrimoniales, fue elemental para el diseño de la ruta turística (Servicio Nacional de Aprendizaje, 2013), dando como resultado guiones interpretativos para los bienes inmuebles patrimoniales. Dado que el guión interpretativo precisa tener muy clara y definida la ruta a seguir, así como los objetivos generales y específicos al realizar tal recorrido (Ministerio de Ambiente de Perú, Dirección General de Educación, Cultura y Ciudadanía Ambiental. 2013), se elaboraron una serie de guiones interpretativos enfocados en resaltar acontecimientos suscitados en la ciudad de Jipijapa durante la etapa republicana del Ecuador (Toala, M. 2012). 


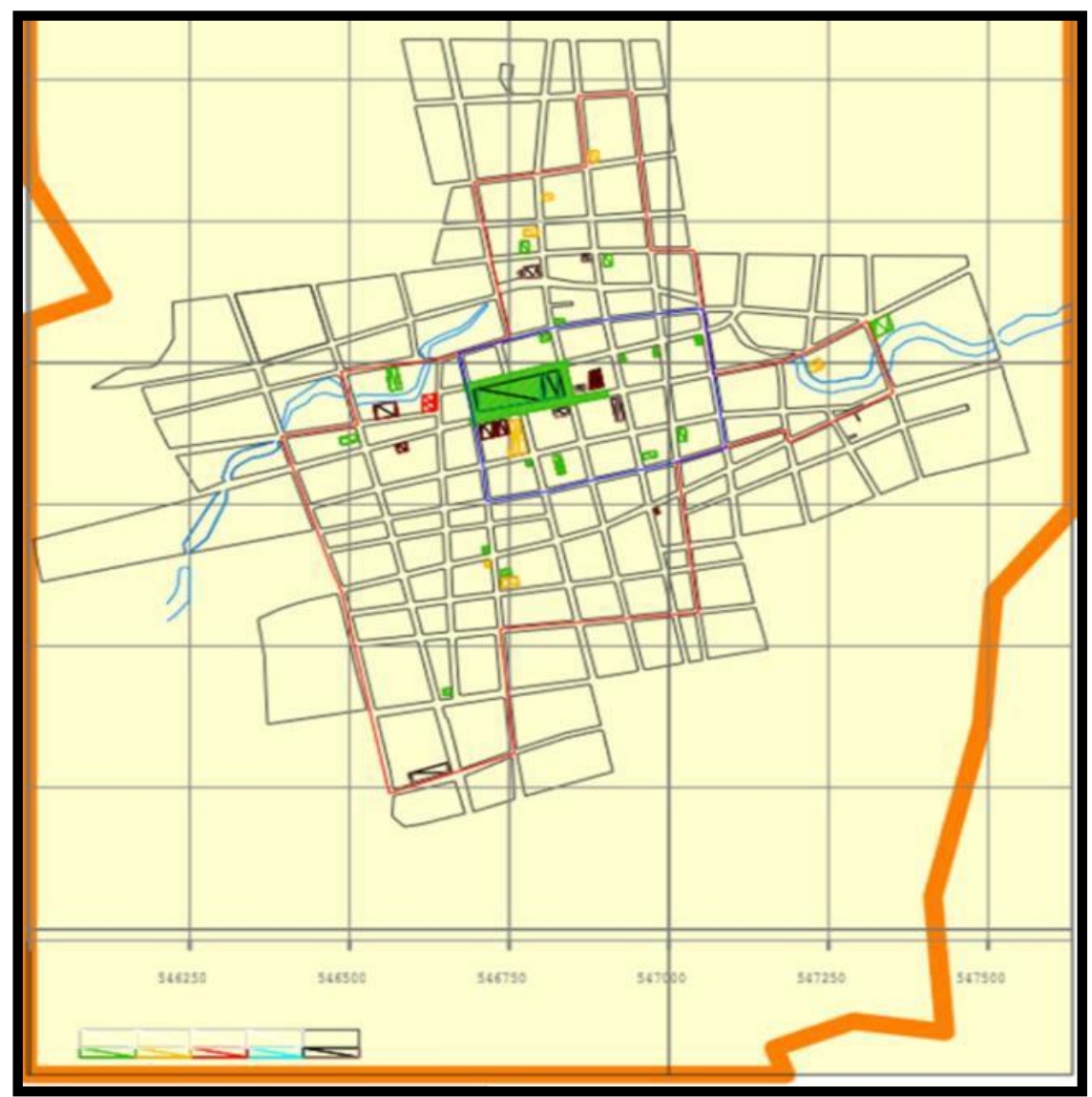

Figura 9. Mapa elaborado por Sr. Diego Solano, Instituto Nacional de Patrimonio Cultural del Ecuador, 2016.

Para el manejo de grupo de visitantes se recurrió a determinar capacidad de carga física, real y efectiva (Cifuentes, M. et al, 1999), la cual estableció un total de 5 grupos diarios, cada uno compuesto por un total recomendado de 20 personas para su correcta operación y manejo de parte de los guías locales. Guías que deberán estar capacitados en procesos de gestión del patrimonio, pudiendo “patrimonializar” el territorio (Guerra, F; Sureda, J; Castells, M. 2011) definiendo los elementos patrimoniales de Jipijapa; "creando" patrimonio y transformando elementos patrimoniales en recursos de atracción turística. En estas dimensiones los guías deberán realizar revisiones constantes al itinerario de la ruta turística patrimonial "Villa de Oro", deberán verificar y comprobar el estado actual de elementos del patrimonio etnológico, elementos del patrimonio histórico y artístico y elementos del patrimonio natural que son utilizados como recursos de atracción turística de la ciudad de Jipijapa. 
Modelo de ruta turística patrimonial para impulsar bienes inmuebles patrimoniales culturales en el desarrollo turístico en el cantón Jipijapa, Ecuador

En este aspecto, la ciudad de Jipijapa cuenta con la aportación realizada desde la academia, con proyectos investigativos - educativos elaborados desde la carrera de turismo de la Universidad Estatal del Sur de Manabí (Pin Figueroa, W. 2015)

\section{Conclusiones}

Las diversas potencialidades culturales, históricas, arquitectónicas y sociales vinculadas al conjunto de bienes inmuebles que integran los denominados valores culturales nacionales de la ciudad de Jipijapa, decretados mediante acuerdo ministerial Nro. DM-2017-019, representan una oportunidad para incorporar a la masa económica regional excedentes dinerarios por concepto de turismo nacional e internacional, producto de la activación de nuevas demandas en las áreas de servicios, comercio, comida, transporte y telecomunicación, entre otros. Se trata pues, de la activación e incremento de los flujos de bienes y servicios, no solo derivados de aquellas actividades que se efectúan en vinculación directa con la ruta turística, sino también aquellos otros surgidos de manera indirecta pero que sirven de apoyo o sustento a la primera.

El diseño e implantación de la ruta turística patrimonial "Villa de Oro", no solo representa un circuito turístico de observación y contacto con las realidades locales de esta región, sino también, un sistema integrado donde se incorporan los elementos físicos patrimoniales, con la necesidad de fortalecer los valores históricos, culturales y sociales que permiten por una parte el reforzamiento como sociedad de quienes participan en la propuesta y por la otra, el empoderamiento y divulgación de aquellos elementos que hacen trascendentes e interesantes para otras culturas aquellas ofertas que conforman las rutas turísticas, sus servicios asociados y áreas de influencia.

El tema de la sustentabilidad hoy día cobra cada vez más importancia en nuestras sociedades, hemos entendido que existe una necesidad creciente en el diseño de nuevas fórmulas que permitan 
desarrollo y crecimiento sin que ello implique el agotamiento irreversible de los recursos. En ese orden de idea, el turismo y en particular el contemplativo, cultural e histórico, representa una opción válida que permite por una parte el bienestar humano a través de las mejoras en la calidad de vida y por la otra la conservación, en este caso de elementos históricos y culturales de la ciudad de Jipijapa.

La declaratoria de los bienes inmuebles que comprenden los valores culturales nacionales de la ciudad de Jipijapa, representan desde el punto de vista jurídico una propuesta orgánica demandante de la motricidad necesaria que permita se cumplan los fines para lo cual fue creado. Son diversas las estrategias y acciones que caben en la esfera de posibilidades fácticas, en ese universo de opciones se encuentra el diseño de la ruta turística patrimonial "Villa de Oro", quien viene a dinamizar y dar vida a esos elementos que han sido protegidos mediante Ley por intermedio de la incorporación de la sociedad de manera activa, generando recursos económicos y a su vez realzando, utilizando y conservando tales valores culturales.

Desde un punto de vista práctico, la propuesta de ruta turística patrimonial, representa una oportunidad para activar la actividad académica y de formación en las áreas vinculadas al comercio, la cultura, historia y el turismo, además ofrece incentivos de diferente naturaleza para la comunidad, fungiendo además como motor de desarrollo, fuente de congresos internacionales de investigación en turismo, patrimonio cultural, hotelería y gastronomía, entre otros.

La ruta turística patrimonial "Villa de Oro" representa para la ciudad un nuevo producto turístico que sin lugar a dudas apuntala un nuevo modelo de gestión del patrimonio que logra enaltecer la identidad cultural de su población, afianzando los valores y saber ancestrales del sitio, a través de la flexibilización de la actual oferta turística de la ciudad. 
Modelo de ruta turística patrimonial para impulsar bienes inmuebles patrimoniales culturales en el desarrollo turístico en el cantón Jipijapa, Ecuador

\section{Bibliografía}

1. Ander-Egg, E. (1993). Técnicas de Investigación Social, Argentina: Magisterio del Río de la Plata.

2. Comité Científico Internacional de Turismo. (22 de 10 de 1999). Carta Internacional Sobre Turismo Cultural La Gestion Del Turismo En Los Sitios Con Patrimonio Significativo. Guadalajara, Mexico: ICOMOS.

3. Fassio, A; Pascual, L. (2015) Apuntes para desarrollar una investigación en el campo de la administración y el análisis organizacional. Ciudad Autónoma de Buenos Aires: Eudeba.

4. Gobierno Provincial de Manabí. (13 de 07 de 2017). Jipijapa. Obtenido de http://www.manabi.gob.ec/cantones/jipijapa

5. Hernández Ramírez, J. (2011). Los caminos del patrimonio. Rutas turísticas e itinerarios culturales. Pasos. Revista de Turismo y Patrimonio Cultural, 9(2): 225-236.

6. H. Congreso Nacional de Ecuador (2004). Ley de Patrimonio Cultural. Registro Oficial 465. Ecuador. ICOMOS. (1976). Carta del Turismo Cultural. Carta del Turismo Cultural. Bruselas: ICOMOS.

7. ICOMOS. (1976). Carta Del Turismo Cultural. Carta Del Turismo Cultural (pág. 3). BRUSELAS: ICOMOS.

8. Inglis, D. y Holmes, M. (2003). Highland and other haunts. Ghosts in Scottish tourism. Annals of Tourism Research, 30(1): 50-63.

9. López-Guzmán, T. y Sánchez, S. (2008). La creación de productos turísticos utilizando rutas enológicas. Pasos. Revista de Turismo y Patrimonio Cultural, 6(2): 159-171.

10. Mackinnon, Child \& Thorsell (1996). Planificación física para el desarrollo turístico. Guia para las mejores prácticas de ecoturismo en Áreas Protegidas. Washington: IUCN.

11. Ministerio de Cultura y Patrimonio del Ecuador (2017) Acuerdo Ministerial Nro. DM - 2017 019. Quito. Ministerio de Cultura y Patrimonio del Ecuador.

12. OMT. (2013). Turismo y Patrimonio cultural inmaterial. Madrid: Organización Mundial de Turismo.

13. Oxinalde, M. d. (2002). Ecoturismo: Nuevas formas de turismo en el espacio rural. Madrid: S.A. BOSCH.

14. Pérez Respaldiz, L. (2014). Turismo Cultural: Una oportunidad para el desarrollo local. Santiago: SERNATUR.

15. Plass W., J. (2012). Importancia del turismo patrimonial. Chile: Organización Mundial de Turismo.

16. Rengifo, J. I. (2006). Rutas culturales y turismo en el contexto español. Boletín del Instituto Andaluz del Patrimonio Histórico, 60: 114-125.

17. Suárez, MR. (2004). Los itinerarios culturales. The CIIC Scientific Magazine. 15-19

18. Toala, M. (2012) Jipijapa...Su historia. Jipijapa -Manabí. Casa de la Cultura Ecuatoriana Núcleo Autónomo de Manabí Eloy Alfaro Delgado.

19. Zeas Sacoto P. (2008) Guía Manual para la elaboración de un plan de prdenamiento ecológico, territorial y de asentamientos humanos (POETAH). Quito. CODEN. 